REVISÃO

\title{
NEMATOIDES: BIOINDICADORES DE SUSTENTABILIDADE E MUDANÇAS EDAFOCLIMÁTICAS ${ }^{1}$
}

\author{
CECÍLIA HELENA SILVINO PRATA RITZINGER ${ }^{2}$, MARILENE FANCELLI², \\ ROGÉRIO RITZINGER ${ }^{3}$
}

RESUMO- Em vista do desconhecimento da importância econômica dos fitonematoides, isoladamente, em cada cultura, esses organismos têm sido frequentemente negligenciados nos agroecossistemas, somente assumindo status de patógeno quando sua população se encontra muito elevada, com prejuízos acentuados. Contudo, somente $10 \%$ do universo de nematoides causam danos às plantas, $25 \%$ são nematoides agrupados nos níveis tróficos de fungívoros ou micófagos, bacterívoros e onívoros, de acordo com o tipo de sua alimentação. Não se conhece a relação do nível populacional do nematoide com o nível de dano nas plantas. Por esse motivo, os defensivos químicos são, em sua maioria, a opção mais usada ou a preferida pelos agricultores, para o manejo, podendo promover o desequilíbrio na comunidade, refletindo em risco ambiental. Para a avaliação de risco, proveniente de substâncias químicas, impactos sobre diferentes tipos de manejo dos solos, bem como distúrbios que eles sofrem, vários testes de toxicidade com nematoides têm sido realizados e há crescente demanda em sua utilização em países mais desenvolvidos. A análise da comunidade presente em determinado ecossistema, de acordo com os hábitos alimentares e o índice de maturidade, provou ser bom indicador a danos causados por poluentes ou distúrbios ecológicos. Nessa revisão, são apresentados dados que demonstram que esses organismos podem ser utilizados de forma satisfatória em estudos de sustentabilidade de ecossistemas, devido à sua abundância, diversidade e respostas à toxicidade e distúrbios ambientais.

Termos para indexação: bacterívoros, fungívoros, onívoros, comunidade de nematoides, abundância, biomassa.

\section{NEMATODES: BIOINDICATOR OF SUSTAINABILITY AND EDAPHOCLIMATIC CHANGES}

\begin{abstract}
Due to their economic importance being unknown in most crops, apart from other pests, plant-parasitic nematodes have been frequently neglected in the agroecosystems, assuming 'status' only when their population is very high, causing accentuated damages which leads to significant yield losses. Nevertheless, only $10 \%$ of the nematode universe is parasites causing damages to plants; $25 \%$ are free-living forms including bacterivorous, fungivorous, omnivorous or predators according to their feeding habits. The relationship between the nematode population level and the damage level for each crop is unknown. Therefore, pesticides are considered the best control option for farmers, but the indiscriminate use could lead to the instability of soil communities resulting in environmental risk. For the risk evaluation, originated from chemical substances, impacts on different types of soil management, as well as disturbances suffered by the soil, several toxicity tests with nematodes have been carried out and there is an increased demand in their use in highly developed countries. The analysis of the soil nematode communities in some ecosystems, according to their feeding habits and maturity index proved to be a good indicator to the damages caused by pollutants or ecological disturbances. In this review, data that demonstrate that these organisms can be used in a satisfactory way in studies of sustainability of ecosystems, due to their abundance, diversity and responses to the toxicity and environmental disturbances, are presented.
\end{abstract}

Index terms: bacterivorous, fungivorous, omnivorous, nematode community, abundance, biomass.

\footnotetext{
1(Trabalho 125-10). Recebido em: 13-05-2010. Aceito para publicação em: 19-10-2010.

${ }^{2}$ Eng. Agr. PhD.., Pesquisador da Embrapa Mandioca e Fruticultura Tropical, Cruz das Almas-BA - e-mail: cecilia@cnpmf.embrapa.br, rogerio@cnpmf.embrapa.br

${ }^{3}$ Eng. Agr. DSc.., Pesquisador da Embrapa Mandioca e Fruticultura Tropical, Cruz das Almas-BA - e-mail: fancelli@enpmf.embrapa.br
} 


\section{INTRODUÇ̃̃O}

Os nematoides constituem um diverso grupo dos invertebrados, abundantes como parasitas ou na forma de vida livre no solo, em ambientes aquáticos ou marinhos. Segundo Barker (1998), existem mais de 15.000 espécies descritas, representando somente uma pequena porção dentro do filo Nematoda. Cerca de $26 \%$ dos gêneros descritos habitam o solo sob diferentes grupos funcionais, bacterívoros, fungívoros, onívoros, predadores ou fitoparasitas. A umidade do solo, a umidade relativa e os fatores ambientais afetam diretamente a sobrevivência dos nematoides. Os nematoides possuem variadas formas de adaptação a mudanças que ocorrem no ambiente causadas por diversos fatores, entre os quais o manejo dos cultivos, estresse climático, época de plantio, fisiologia das plantas e melhoramento genético (BLAKELY et al. 2002).

A dormência ou diapausa constitui importante fator para a sobrevivência e longevidade de muitas espécies sob diferentes condições climáticas, onde a mudança de sexo e a taxa de reprodução podem melhorar de sobremaneira a sobrevivência de novas gerações. A agregação, ou formação da larva 'dauer', confere relativa resistência às diferentes variações climáticas. Outros nematoides, sob estresse ambiental, podem sobreviver em estado de quiescência temporária e entrar em anidrobiose ou outros estados extremos que permitem sua prolongada sobrevivência. Uma proporção substancial de nematoides possui esses estádios inativos para sobrevivência diante de condições adversas (McSORLEY, 2003). Essa capacidade de sobrevivência dos nematoides é de extrema importância para entender fenômenos de epidemias e dispersão de nematoides sob condições de adversidade ambiental e vegetal.

Para entender os processos que ocorrem no solo, devido ao manejo adotado ou a reflexos oriundos das mudanças climáticas na restauração dos solos, a estrutura da teia alimentar do solo é uma ferramenta completa que pode auxiliar a identificar o ecossistema do solo. Porém, poucas estratégias são orientadas para utilização dessas informações. Trabalhos realizados por Biederman et al. (2008) revelam a importância do monitoramento da população de nematoides ao longo das estações do ano e do ciclo das plantas. Esses autores relatam comportamentos diversos sob diferentes situações em diferentes ecossistemas. Outras informações, como densidade, diversidade, riqueza de espécies, índice de maturidade, estrutura dos solos e presença de fitonematoides podem variar ao longo do tempo.

A existência de grupos funcionais, de acordo com a alimentação, e as estratégias reprodutivas permitem seu uso como indicadores de condição de uso da terra. Desta forma, alterações climáticas em temperatura e $\mathrm{CO}_{2}$ influenciam na distribuição de alimentos para esses grupos. Então, a identificação desses indivíduos por espécie em cada grupo funcional é necessária e permite avanços no entendimento de processos oriundos das mudanças climáticas, resiliência do ecossistema (YEATES, 2003).

Assim, por meio desses estudos, pode-se obter uma previsão de comportamento na cadeia alimentar. Por exemplo, a presença de resíduos na superfície dos solos pode afetar indiretamente a estrutura da comunidade de nematoides por promover maior densidade de raízes, maior concentração de matéria orgânica, exsudados radiculares, favorecendo grupos de nematoides oportunistas, que se beneficiam e respondem de maneira rápida pela oferta de alimento (BORGES et al., 2003). Portanto, com o aumento da temperatura, a atividade microbiana em sistema rico em matéria orgânica responde de forma diferente aos ambientes com menor teor de matéria orgânica e temperaturas elevadas (RITZINGER; FANCELLI, $2006 \mathrm{a}, \mathrm{b}$ ).

Com a elevação de temperatura, outra resposta fisiológica, particularmente sensível à redução do potencial da água nas plantas, é a alteração da síntese proteica. Como consequência, algumas enzimas que apresentam alta relação síntese-degradação, como, por exemplo, a redutase do nitrato, exibem forte inibição à redução do potencial hídrico. Segundo Viglierchio (1991), os órgãos sensoriais do nematoide são os responsáveis pelas diferentes reações aos diferentes estímulos bióticos e abióticos. As estruturas sensoriais são responsáveis pelas diferentes respostas e comportamentos, pois estão ligadas diretamente ao sistema nervoso do nematoide, gânglios ventrais e dorsais. Entre elas, as papilas cefálicas, labiais, os cefalídios, deirídios e fasmídios são responsáveis pelo envio de informações que modificam o comportamento do nematoide para sua sobrevivência (HUETTEL, 1986). Nesse sentido, mudanças de comportamento da planta com relação a $\mathrm{CO}_{2}$ afetam a fotossíntese da planta da mesma forma que a presença do nematoide afeta a produção de hormônios produzidos pela planta como citoquininas e giberelinas que, consequentemente, modificam a translocação de água e modificam a abertura e o fechamento dos estômatos (WALLACE, 1987).

Segundo Viglierchio (1991), há outros órgãos sensoriais que ainda não possuem uma identificação correta de sua função. Em alguns nematoides, já 
foi identificada alteração de seu comportamento na presença de $\mathrm{CO}_{2}$, a depender de sua concentração. Geralmente, sob alta concentração, a movimentação do nematoide é reduzida e, ao contrário, sob baixa concentração de $\mathrm{CO}_{2}$, a resposta aumenta. Contudo, poucos estudos têm sido feitos por nematologistas para entendimento dessas influências no processo de sobrevivência ou adaptação do nematoide.

$\mathrm{O}$ binômio temperatura e $\mathrm{CO}_{2}$, certamente, muda a fisiologia da planta. Assim como o metabolismo primário das plantas pode ser modificado em resposta a fatores abióticos, o metabolismo secundário também é influenciado, levando à diminuição de uma variedade de compostos. Consequentemente, a adaptação do nematoide, por meio de mudança de seu metabolismo, por exemplo, constitui ferramenta para sua longevidade mediante essas modificações.

Estudos realizados por Li et al. (2009), utilizando a técnica de enriquecimento de $\mathrm{CO}_{2}$ (FACE), para avaliar o comportamento de nematoides sob incorporação de resíduos e adubação nitrogenada, revela que a sazonalidade é um importante fator para determinar as mudanças em abundância e diversidade. O efeito da adição de resíduos foi mais evidente que a elevação de $\mathrm{CO}_{2}$. A comunidade de nematoides no solo e os índices ecológicos foram significativamente afetados pela interação da elevação de $\mathrm{CO}_{2}$ e com a aplicação de resíduos e adubação nitrogenada. Esse efeito foi observado pela abundância de onívoros-carnívoros e nematoides totais. A adição de resíduos estimula o índice estrutural e inibe a resposta dos fitoparasitas sob elevada concentração de $\mathrm{CO}_{2}$.

$\mathrm{O}$ solo oferece várias formas de proteção à desidratação dos nematoides. Os nematoides que vivem dentro das raízes ou dentro de insetos possuem privilégio de proteção ótima à dissecação enquanto a saúde desses hospedeiros persistir. Contudo, os nematoides ou estágios dos nematoides que não vivem dentro do hospedeiro (raízes ou insetos) são protegidos pela umidade do solo, mas ficam sujeitos à desidratação mais rápida, enquanto esse solo perde umidade, seja por meio de aumento da temperatura, seja por meio do manejo (CHRISTIE, 1959). Esse risco aumenta na interface com ar-solo (WOMERSLEY, 1987).

No Brasil, poucos estudos são realizados com o comportamento de fitonematoides a variações edafoclimáticas. Estudos realizados por Asmus e Ishimi (2009) sobre a flutuação populacional de Rotylenchulus reniformis, revelam que a densidade e a composição populacional de $R$. reniformis são influenciadas pela presença de plantas de algodoeiro. Contudo, a abundância da população de $R$. reniformis é influenciada diretamente pela temperatura do solo.
Nesse estudo, dentro das variáveis edafoclimáticas avaliadas, a precipitação e a umidade do solo pouco influenciaram sobre a população de $R$. reniformis.

\section{CICLO DE VIDA}

O ciclo de vida do nematoide envolve o ovo, quatro estádios juvenis, J1, J2, J3 e J4 e o estádio adulto. Em algumas espécies, o estádio juvenil assemelha-se ao estádio adulto, podendo dificultar a identificação das fases; mas, na grande maioria do grupo, existe uma diversidade entre cada estádio (WHARTON, 1986). O ciclo de vida de alguns nematoides, oferece grande oportunidade de resistência ao estresse ambiental, como a formação de cisto que abriga a massa de ovos de algumas espécies. Outros nematoides, por ocasião do crescimento e desenvolvimento, efetuam a primeira troca de cutícula ainda dentro do ovo, mantendo a cutícula trocada aderida à nova cutícula como uma proteção, até que a segunda troca ocorra, desenvolvendo então o fenômeno da diapausa ou dormência, até que o segundo estádio ocorra. Esse é um mecanismo de sobrevivência que ocorre em estações frias e, geralmente, na ausência de hospedeiro. A diapausa e o desenvolvimento da dormência em nematoides parece que ocorrem mais no estádio de ovo e no estádio juvenil, dentro do ovo.

A membrana semipermeável serve como barreira e, por si só, confere proteção ao nematoide. Permite a entrada seletiva de pequenas moléculas ou íons e inibe a passagem de íons maiores, como pectinas e proteínas (VIGLIERCHIO, 1991). Dessa forma, por meio dessa seletividade natural, sob estresse, o nematoide possui habilidade de prolongar seu ciclo de vida, da mesma forma que sob condições ótimas pode encurtar as fases de desenvolvimento, tendo seu ciclo mais curto, originando mais gerações por ano.

Estudos têm demonstrado que, sob condições de baixa temperatura, congelamento lento, o nematoide pode formar cristais de gelo e ter suas membranas ou organelas destruídas. Contudo, quando o congelamento é procedido por meio de agentes protetores e utilização de nitrogênio líquido, sua sobrevivência pode durar anos (SASSER; CARTER, 1985).

Em Meloidogyne naasi Franklin, a eclosão da larva, estimulada sob condições de baixas temperaturas, induz o fenômeno da diapausa. Em algumas espécies de Meloidogyne spp. e nematoides de cisto (Heterodera spp. e Globodera spp.), somente uma porção das larvas eclode rapidamente, enquanto a outra porção eclode lentamente no tempo. Esse fenômeno tem sido reconhecido por diversos cientistas 
(EVANS, 1987; GUIRAN, 1979; VAN GUNDY, 1985; ZHENG; FERRIS 1991; HUANG; PEREIRA, 1994; SIKORA; NOEL, 1996; YEN et al. 1995). O estudo da eclosão da larva é complexo. Algumas larvas eclodem rapidamente em água, outras necessitam de estímulos sob a forma de exsudados de raízes hospedeiras para eclodir, enquanto outras eclodem lentamente, mesmo sob a presença de água ou exsudados. Portanto, o estímulo da eclosão da larva e o término da dormência em várias espécies estão altamente influenciados pela temperatura, presença de hospedeiro ou ainda exsudados de raízes. Nesse caso, a liberação de exsudados de raízes depende basicamente da cultivar, da fenologia e de outros fatores.

\section{REPRODUÇÃO}

A taxa sexual entre as espécies é determinada, em sua maioria, por condições ambientais. A reprodução por meio de machos e fêmeas promove maior diversidade entre as espécies, embora a reprodução por partenogênese possa também ocorrer.

A produção de machos tem sido muito estudada no nematoide-das-galhas. A larva eclode do ovo no estádio $\mathrm{J}_{2}$ e migra no solo até penetrar no tecido da raiz do hospedeiro quando então estabelece o sítio de infeção. Quando o estádio $\mathrm{J}_{2}$ começa a alimentar-se, torna-se imóvel, aumenta de tamanho e espessura, e passa por diferentes trocas de cutícula, desenvolvendo-se uma fêmea que se reproduz por partenogênese (CHRISTIE, 1959). Machos podem ser raros na população, mas, em alguns casos, podem chegar a $60 \%$. Esse fato tem sido atribuído ao estresse sofrido pelo nematoide, seja por deficiência nutricional da planta hospedeira, redução na taxa fotossintética, idade da planta, seja por reguladores ou inibidores de crescimento da planta, aumento da densidade populacional do nematoide, presença de outros patógenos, nível de resistência da planta hospedeira, temperatura e irradiação. Segundo Triantaphyllou (1973) e Papadopoulou e Triantaphyllou (1982), se o estresse ocorre durante o desenvolvimento, a fêmea, no segundo estádio juvenil, pode reverter seu desenvolvimento, modificando sua sexualidade (intersexo ou macho). A fertilização dos ovos traz muitos benefícios, além da maior chance de sobrevivência nas espécies. Contudo, a forma móvel do macho e a possibilidade de locomover-se a outras áreas, uma vez que a fêmea é imóvel, sobre condições de estresse, pode ser uma das maiores vantagens de sobrevivência e adaptação no gênero Meloidogyne (BIRD, 1971).
Entre as respostas ao estresse ambiental, diferentes estádios de desenvolvimento surgem com a diminuição do metabolismo do nematoide, conferindo-lhe uma proteção às condições de estresse, como maior sobrevivência e adaptação rápida à condição de estresse. Alguns nematoides podem sobreviver sob baixa condição de $\mathrm{O}_{2}$, passando então da condição aeróbia para anaeróbia (BIRD, 1971; RUESS et al., 1999).

Sabe-se que a habilidade da 'dauer' larva a resistir ao estresse ambiental e retornar ao estágio normal, varia de espécie para espécie. A agregação do nematoide e a diminuição de seu metabolismo confere-lhe proteção contra a dissecação e permite sua sobrevivência sobre condições adversas. Nesse fenômeno, alguns nematoides podem adaptar-se a condições de baixa umidade.

\section{RESPOSTA A EXTREMOS AMBIENTAIS}

O nematoide pode sobreviver a condições prolongadas de falta de alimento por diferentes mecanismos. A longevidade é garantida devido à redução de seu metabolismo e, em algumas condições, por meio de armazenamento de lipídios ou glicose. Segundo Viglierchio (1991), a larva de Meloidogyne recém-eclodida pode sobreviver sob condições ótimas de temperatura por mais de 30 dias. Portanto, o mecanismo de sobrevivência varia grandemente em cada espécie e para cada situação, seja por meio de mudanças de temperatura, umidade, fotoperíodo, seja por mudanças nutricionais decorrentes do hospedeiro, presença de predadores, ou outros microrganismos. O melhor desenvolvimento do nematoide, alimentação, migração e reprodução, ocorre entretanto sob condições ótimas de temperatura, acima da qual a deterioração de enzimas e disfunção de algumas membranas afetam seu comportamento. Dentre alguns mecanismos, a quiescência e a diapausa caracterizam o estádio de mudança de seu metabolismo (BIRD; BIRD, 1991; RIDDLE et al., 1987).

Quiescência refere-se ao estádio de dormência, no qual o metabolismo e a atividade do nematoide diminuem diante do estresse ambiental. Diferentemente da diapausa, o estado de dormência termina quando as condições de estresse terminam e o nematoide retorna então a sua atividade.

Em casos de prolongada quiescência, a taxa de metabolismo pode cair a nível tão baixo que parece cessar. Essa extrema condição é referida como anabiose, por Wharton (1986), ou criptobiose, por Cooper et al. (1971). A terminologia anidrobiose é usada frequentemente para definir perda gradual de 
água que ocorre devido ao estresse. Com a redução de água, a atividade do nematoide pode cair de 75$80 \%$ a $2-5 \%$ na forma de anidrobiose (COOPER et al., 1971; NICHOLAS; STEWART, 1989; WHARTON; BARCLAY, 1993). Esse mecanismo favorece o prolongamento e a sobrevivência de espécies a condições de estresse ambiental. Assim, em ambientes onde essas espécies permanecem nessa condição, seja por perda de água no solo devido à evaporação, seja por meio da exposição de solos a temperatura elevadas, essas espécies representam bom indicativo das mudanças climáticas.

Segundo Barret (1991), a reidratação ocorre gradualmente, mas a ocorrência de anidrobiose em vários ciclos do nematoide pode diminuir sua viabilidade. Assim, sugere-se que a extinção de espécies também possa ocorrer no caso de frequentes estresses hídricos, devido à evaporação de água dos solos submetidos a altas temperaturas. O mecanismo responsável pela anidrobiose não é bem conhecido, mas sabe-se que a permeabilidade da cutícula do nematoide diminui, e uma condensação ou empacotamento de tecidos e organelas é observado por ocasião de anidrobiose. Outras modificações são observadas, como o encurvamento do nematoide, mas esse fato é observado também na ausência de $0_{2}$ (COOPER et al., 1971).

Segundo Womersley (1987), a estratégia de sobrevivência é utilizada na natureza para permitir a longevidade dos nematoides. Muitas vezes, esse fato pode passar despercebido no processo de extração de nematoides, pela ausência do nematoide na amostra (McSORLEY, 1987). Por esse motivo, Freckman et al. (1977) sugerem métodos de extração mais acurados. No caso de Pratylenchus penetrans, segundo Townshend (1984), quando o solo seca rapidamente, $22-31 \%$ da população fica no estado de anidrobiose, e quando o solo seca lentamente, essa condição passa para 58-70\%. Pratylenchus penetrans e $R$. reniformis que habitam solo e raízes podem sobreviver no estádio de anidrobiose, por um curto espaço de tempo quando comparados com outros nematoides da parte aérea da planta (TOWNSHEND, 1984; WOMERSLEY; CHING, 1989).

Os nematoides que possuem reprodução sexuada podem, sob condições de estresse, originar uma geração mais resistente ou adaptada às novas condições submetidas. Alguns respondem mais prontamente ao aumento da temperatura, enquanto outros, ao estresse hídrico ou à aeração. Contudo, a temperatura constitui fator principal para determinar a embriogênese, formação do ovo e eclosão da larva. Em sua maioria, possuindo faixa ideal de reprodução aos $25^{\circ} \mathrm{C}$ (BARKER et al., 1985), podem, conforme a espécie, ser denominados termófilos (não sobrevivem sob temperaturas abaixo de $10{ }^{\circ} \mathrm{C}$, como M. incognita, M. javanica) ou criófilos (podem sobreviver a temperaturas abaixo de ${ }^{0} \mathrm{C}$, como, por exemplo, M. hapla).

Sob condições de seca, o estado de anidrobiose é mais comum para os nematoides que sobrevivem na parte aérea da planta. Contudo, na interface solo-ar, devido à porosidade do solo, esse fenômeno também ocorre. Segundo McSorley (2003), o entendimento da ecologia dos nematoides em ambientes adversos permanecerá desconhecida se a porção de nematoides em estado de anidrobiose for subestimada. Portanto, os estudos em ambientes marginais ou sob estresse devem continuar para agregar mais informações sobre formas de sobrevivência de nematóides sob condições adversas.

\section{CONCLUSÃO}

1-O ambiente pode oferecer uma serie de obstáculos à sobrevivência e longevidade dos nematoides. Assim, a abundância e a diversidade de nematoides pode alterar-se sob as variadas mudanças ambientais (pela ação do homem ou da natureza). Modificações físicas, químicas, atributos biológicos do ecossistema, como irrigação, práticas de cultivo, drenagem, utilização de pesticidas, monocultivos ou policultivos, podem resultar da diminuição de nematoides nativos (níveis não detectáveis) como resultar na introdução ou desenvolvimento rápido de outra espécie nativa ou introduzida a níveis elevadíssimos (YEATES, 1999).

2-A amostragem para análise da comunidade de nematoides deve ser na escala temporal e espacial. Devido a essa diversidade, o relacionamento entre grupos funcionais de nematoides e os processos ecológicos decorrentes têm constituído, portanto, uma ferramenta para estudos em campos experimentais. Alguns experimentos têm demonstrado que fitonematoides aumentam o fluxo de carbono das raízes para a biomassa microbiana do solo. A diversidade tende a ser maior em ecossistemas que sofrem menor distúrbio. Segundo Yeates (1999, 2003), o índice da fauna de nematoides reflete as mudanças que ocorrem na comunidade devido aos processos ecológicos que acontecem com as mudanças no ecossistema.

3-Diferentes graus de quiescência, por meio da diminuição de metabolismo, particularmente a anidrobiose, permitem a sobrevivência de nematoides a condições de extrema variação de temperatura, ausência de hospedeiros, restos culturais ou mesmo 
sua dispersão por meio de poeira, sobretudo em condições de fortes vendavais.

4 - Na ação do projeto CLIMAPEST, desenvolvido pela Embrapa Meio Ambiente, impactos de mudanças climáticas sobre doenças, pragas e plantas daninhas, o levantamento de populações de fitonematoides e nematóides de vida livre, a cada ciclo da cultura, sob diferentes concentrações de $\mathrm{CO}_{2}$ atmosférico e de alterações da temperatura sobre problemas fitossanitários, em frutas tropicais, em condições de estufa de topo aberto, permitirão melhor entendimento de seu comportamento dentro do agroecossistema, por meio da análise e correlações entre as populações e a temperatura e/ou $\mathrm{CO}_{2}$ dentre outras possíveis variáveis. O monitoramento desses nematoides sob os diferentes tratamentos e cultivos, com ou sem OTC ('Open Top Chambers'), serve como indicativo do impacto das mudanças climáticas. Os índices das comunidades de nematoides de vida livre no solo (taxa de ocorrência, abundância e diversidade) são essenciais para detectar impactos sobre diferentes tipos de manejo dos solos, bem como distúrbios que eles sofrem.

\section{REFERÊNCIAS}

ASMUS, G. L.; ISHIMI, C. M. Flutuação populacional de Rotylenchulus reniformis em solo cultivado com algodoeiro. Pesquisa Agropecuária Brasileira, Brasília, v. 44, n. 1, p. 359-372, 2009.

BORGES, A. L.; FANCELLI, M.; RITZINGER, C. H. S. P. Aspectos gerais da produção orgânica de frutas. 2003. In: STRINGHETA, P. C.; MUNIZ, J. N. Alimentos orgânicos: produção, tecnologia e certificação. Viçosa: UFV, 2003. p. 235-288.

BARKER, K. R. Introduction and synopsis of advancements in nematology. In: BARKER, K.R.; PEDERSON, G. A.; WINDHAM, G. L. Plant and nematode interactions. Madison: American Society of Agronomy, 1998. p. 1-20.

BARKER, K. R.; CARTER, C. C; SASSER, J. N. An advanced treatise on Meloidogyne: methodology. Raleigh: North Caroline University, 1985. v.2, 223p.

BARRETT, J. Anhydrobiotic nematodes. In: EVANS, K. (Ed.). Agricultural zoology reviews. Andover: Intercept, 1991. v.4, p.161-176.
BIEDERMAN, L. A.; BOUTTON, T. W.; WHISENANT, S. G. Nematode community development early in ecological restoration: the role of organic amendments. Soil Biology \& Biochemistry, Elmsford, v. 40, p. 2366-2374, 2008.

BIRD, A. F. Specialized adaptations of nematodes to parasitism. In: ZUCKERMAN, B. M.; MAI, W. F.; ROHDE, R. A. (Ed.). Plant parasitic nematodes. New York: Academic Press, 1971. v.2, p.35-49.

BIRD, A. F.; BIRD, J. The structure of nematodes. 2. ed. San Diego: Academic Press, 1991. 316p.

BLAKELY, J.K.; NEHER, D. A.; SPONGBERG, A L. Soil invertebrate and microbial communities, and decomposition as indicators of polycyclic aromatic hydrocarbon contamination. Applied Soil Ecology, Amsterdam, v.21, p. 71-88, 2002.

CHRISTIE, J. R. Plant nematodes their bionomics and control. Gainesville: Agricultural Experiment Stations, 1959.

COOPER JR.; A. F.; VAN GUNDY, S. D. Senescence, quiescence, and cryptobiosis. p. 297-318. In: ZUCKERMAN, B. M.; MAI, W. F.; ROHDE, R. A. (Ed.). Plant parasitic nematodes. New York: Academic Press, 1971. v.2, p.279-3181.

EVANS, A. A. F. Diapause in nematodes as a survival strategy.. In: VEECH, J. A.; DICKSON, D. W. (Eds.). Vistas on nematology. Hyattsville: Society of Nematologists, 1987. p. 180-195

FRECKMAN, D. W.; KAPLAN, D. T.; VAN GUNDY, S. D. A comparison of techniques for extraction and study of anhydrobiotic nematodes from dry soils. Journal of Nematology, Hanover, v. 9, p. 176-181, 1977.

GUIRAN, G. de. A necessary diapause in root-knot nematodes. Observations on its distribution and inheritance in Meloidogyne incognita. Revue de Nematologie, Bondy, v. 2, p. 223-231, 1979.

HUANG, S. P.; PEREIRA, A. C. Influence of inoculum density, host, and low temperature period on delayed hatch of Meloidogyne javanica eggs. Journal of Nematology, Hanover, v.26, p.72-75, 1994. 
HUETTEL, R.N. Chemical communicators in nematodes. Journal of Nematology, Hanover, v.18, p.3-8, 1986.

LI, Q.; CHONGGANG, X.; LIANG, W.; et al. Residue incorporation and $\mathrm{n}$ fertilization affect the response of soil nematodes to the elevated $\mathrm{CO}_{2}$ in a Chinese wheat field. Soil Biology and Biochemistry, Exceter, v.41, p.1497-1503, 2009.

MCSORLEY, R. Extraction of nematodes and sampling methods. In: BROWN, R.H.; KERRY, B.R. (Ed.). Principles and practice of nematode control in crops. Sydney: Academic Press, 1987. p. 13-47.

MCSORLEY, R. Adaptations of nematodes to environmental extremes. Florida Entomologist, Gainesville, v. 86, n. 2, p. 138-142, 2003.

NICHOLAS, W. L.; STEWART, A. C. Experiments on anhydrobiosis in Acrobeloides nanus. Nematropica, DeLeon, v.35, p.489-491, 1989.

PAPADOPOULOU, J.; TRIANTAPHYLLOU, A. C. Sex differentiation in Meloidogyne incognita and anatomical evidence of sex reversal. Journal of Nematology, Hanover, v. 14, p. 549-566, 1982.

RIDDLE, D. L.; GOLDEN, J. W.; ALBERT, P. S. Role of dauer larvae in survival of Caenorhabditis elegans. In: VEECH, J.A.; DICKSON, N.W. (Ed.). Vistas on nematology. Hyatsville: Society of Nematologists, 1987. p. 174-179.

RITZINGER, C. H. S. P.; FANCELLI, M. Matéria orgânica e o manejo integrado de nematoides. In: SIMPÓSIO BRASILEIRO SOBRE BANANICULTURA, 6., 2004, Joinville, SC. Sistemas alternativos de produção... Itajaí: $\mathrm{SBF} /$ ACAFRUTA, 2006a. p. 92-105.

RITZINGER, C. H. S. P.; FANCELLI, M. Manejo integrado de nematoides na cultura da bananeira. Revista Brasileira de Fruticultura, Jaboticabal. v. 28, n.2, p.331-338, 2006 b.

RUESS, L.; MICHELSEN, A.; JONASSON, S. Simulated climate change in subarctic soils: responses in nematode species composition and dominance structure. Nematology, Leiden, v. 1, n. 5, p. 513526. 1999.
SASSER, J. N.; CARTER, C. C. An advanced treatise on Meloidogyne. In: SASSER, J. N.; CARTER, C. C. (Ed.). Biology and control. Raleigh: North Caroline University, 1985. 422p.

SIKORA, E. J.; NOEL, G. R. Hatch and emergence of Heterodera glycines in root leachate from resistant and susceptible soybean cultivars. Journal of Nematology, Hanover, v. 28, p. 501-509, 1996.

TOWNSHEND, J. L. Anhydrobiosis in Pratylenchus penetrans. Journal of Nematology, Hanover, v. 16, p. 282-289, 1984.

VAN GUNDY, S.D. Ecology of Meloidogyne spp. Emphasis on environmental factors affecting survival and pathogenicity. In: SASSER, J.N.; CARTER, C.C. (Ed.). An advanced treatise on Meloidogyne: biology and control. Raleigh: North Carolina State University Graphics, 1985. p.177-182.

VIGLIERCHIO, D. R. The world of nematodes: a fascinating component of the animal kingdom. Davis: University of California, 1991. 266p.

TRIANTAPHYLLOU, A. C. Environmental sex differentiation of nematodes in relation to pest management. Annual Review Phytopathology, Palo Alto, v. 11, p. 441-462, 1973.

WALLACE, H. R. Effects of nematode parasites on photosynthesis. In: VEECH, J.A.; DICKSON, N. W. (Ed.). Vistas on nematology. Hyatsville: Society of Nematologists, 1987. p.253-259.

WHARTON, D. A. A functional biology of nematodes. Baltimore: The Johns Hopkins University Press, 1986.

WHARTON, D. A.; BARCLAY, S. Anhydrobiosis in the free-living antarctic nematode Panagrolaimus davidi (Nematoda: Rhabditida). Fundamental and Applied Nematology, Paris, v. 16, p. 17-22, 1993.

WOMERSLEY, C. A reevaluation of strategies employed by nematode anhydrobiotes in relation to their natural environment. In: VEECH, J.A.; DICKSON, D.W. (Ed.). Vistas on nematology. Hyattsville: Society of Nematologists, 1987. p.165173. 
WOMERSLEY, C.; CHING, C. Natural dehydration regimes as prerequisite for the successful induction of anhydrobiosis in the nematode Rotylenchulus reniformis. Journal of Experimental Biology, Cambridge, v.143, p. 359-372, 1989.

YEATES, G. W. Effects of plants on nematode community structure. Annual Review Phytopathology, Palo Alto, v. 37, p. 127-149, 1999.
YEATES, G. W. Nematodes as soil indicators: functional and biodiversity aspects. Biology and Fertility of Soils, Berlin, v. 37, p. 1999-2010, 2003.

YEN, J. H.; NIBLACK, T. L.; WIEBOLD, W. J. Dormancy of Heterodera glycines in Missouri. Journal of Nematology, Hanover, v. 27, p. 153-163, 1995.

ZHENG, L.; FERRIS, H. Four types of dormancy exhibited by eggs of Heterodera schachtii. Revue de Nematologie, Bondy, v. 14, p. 419-426, 1991. 